\title{
Aspectos Neuropsicológicos en Pacientes Diagnosticados de Tumores Cerebrales
}

\section{Neuropsychologic Aspects in Patients with Brain Tumors}

\author{
Ana Sanz Cortés \\ Fundación Investigación Hospital Clínico San Carlos de Madrid, España \\ $\mathrm{M}^{\mathrm{a}}$ Eugenia Olivares Crespo \\ Hospital Clínico San Carlos de Madrid, España \\ Universidad Complutense de Madrid, España
}

Resumen. El presente estudio pretende definir el papel del tumor en el rendimiento neuropsicológico de pacientes con sospecha radiológica de glioma, siendo la valoración en el momento inicial de la enfermedad, previo a la resección quirúrgica. La muestra incluyó 29 pacientes (18 hombres y 11 mujeres) con una edad media de 54 años. A todos ellos se les ha aplicado una batería neuropsicológica para valorar atención, memoria, habilidades visoconstructivas, organización visual, lenguaje y funciones ejecutivas. Los resultados arrojan que más del $50 \%$ de la muestra, presenta puntuaciones centiles por debajo de 40 o por debajo del punto de corte establecido, para todas las variables, excepto en orientación y recuerdo verbal a corto plazo. El grupo de pacientes con gliomas de bajo grado presenta un mejor rendimiento en habilidades de planificación y seguimiento de una estrategia determinada, con respecto a los pacientes con glioma de alto grado. No se observan diferencias en el rendimiento neuropsicológico en relación al hemisferio afectado. Además, dado que la edad es una variable que influye en el rendimiento neuropsicológico, se identifico que ésta era la encargada de explicar las diferencias entre pacientes con glioma de alto y bajo grado, no siendo así en cuanto al hemisferio afectado.

Palabras clave: glioma, valoración neuropsicológica, déficit neuropsicológico.

\begin{abstract}
The aim of this study was to define the role of a glioma in neuropsychological performance, in the initial stage of the disease. Materials and Method: The sample included 29 patients with radiological suspected glioma who were undergoing surgical resection (18 men and 11 women) with a mean age of 54. All subjects were administered a neuropsychological battery assessing attention, memory, language, visuoconstructive skills, visual organization, language and executive functions. The results show that over $50 \%$ of the sample presented scores below the 40 percentile or below the cut-off for all variables, except for orientation and short-term verbal memory. In addition, patients with low grade gliomas showed better performance in planning and monitoring skills of a particular strategy, with respect to patients with light grade glioma. There were no differences in neuropsychological performance in relation to the hemisphere affected by the tumor. Mainly, the age is the variable responsible for explaining differences in cognitive performance of patients studied. Keywords: glioma, neuropsychological assessment, neuropsychological deficit.
\end{abstract}

La correspondencia sobre este artículo debe enviarse a la primera autora al e-mail:ana.sanz@cop.es 


\section{Introducción}

En el campo de la Neurooncología es necesario considerar que el término de tumor cerebral engloba un amplio número de procesos neoplásicos con diferencias histológicas, incluyendo las metástasis de tumores sistémicos (Peterson, 2001). Los tumores primarios del Sistema Nervioso Central (SNC) representan el $2 \%$ de todas las neoplasias en población adulta y, de forma específica, las lesiones más frecuentes son los gliomas, que constituyen el 50\% de todos ellos (Levin y Kyristis, 2000), dividiéndose en gliomas de bajo grado (grado I y II) y de alto grado (III y IV).

En el informe anual mundial del 2008 (Bondy y cols., 2008), se estima que la incidencia de los tumores cerebrales malignos, en función del género, es de $3.7 / 100000$ en hombres y 2.6/100000 en mujeres; siendo estas cifras en España de 8.7 y 5.4 respectivamente.

La Organización Mundial de la Salud estima que la supervivencia media de los pacientes con gliomas varía desde los diez o doce meses para tumores de grado IV hasta los siete años para los de grado I, en función de la histología; varían también de acuerdo a la edad del paciente y los tratamientos recibidos (Molina y cols., 2001). En general, los gliomas son responsables de la pérdida del $7 \%$ de años de vida de la población menor de 70 años (Afra, Muller y Benoist, 1978); registrándose 2631 casos de fallecidos por tumores del SNC en España en el año 2007 (www.seom.org).

De acuerdo al comprometido pronóstico de esta patología, el objetivo principal de los tratamientos oncológicos es potenciar los métodos y técnicas que mejoran la calidad de vida a corto y medio plazo, así como, que consigan minimizar la neurotoxicidad en los pacientes afectos. En este sentido, uno de los factores más relevantes en el estudio de la calidad de vida en esta población, es el funcionamiento neuropsicológico, principalmente por el impacto específico de la localización tumoral en las diferentes áreas de la vida de los pacientes, y por su papel predictor en la progresión de la enfermedad (Meyers y Hess,
2003). Además, es necesario considerar la necesidad de un adecuado funcionamiento neuropsicológico para facilitar información sobre los síntomas y cumplimentar el tratamiento prescrito.

En uno de los primeros estudios de revisión en esta área de conocimiento (Taphoorn, 2003), se informa que el $90 \%$ de los pacientes diagnosticados de gliomas presentan crisis epilépticas, déficits cognitivos objetivos y quejas subjetivas, que incluyen problemas en memoria, atención, funciones ejecutivas y lenguaje. Además, se señala la presencia de deterioro cognitivo en personas con glioma de bajo grado, aunque no haya progresión de la enfermedad. Así, Taphoorn (2003) y Arrieta y García (2009) enumeran una serie de factores que correlacionan con dicho deterioro cognitivo, tales como: tumor inicial, tratamientos oncológicos recibidos, aplicación de fármacos para el control de la epilepsia, malestar psicológico y la combinación de todos ellos. Asimismo, hay autores que citan otras variables que influyen en el rendimiento neuropsicológico, dando especial importancia a la edad (Perea y Ladera, 1995).

Los estudios en el área de la Neurooncología presentan una serie de limitaciones metodológicas referidas al diseño de los mismos, tamaño y heterogeneidad muestral, así como al momento de medida y los instrumentos neuropsicológicos aplicados.

En relación al diseño, la mayor parte de estudios son de naturaleza transversal, esta limitación es debida a la complejidad en la recogida de datos en esta población. En los estudios prospectivos, el tamaño de la muestra es pequeño debido a una elevada mortandad experimental, así como, clínica. Además, en la mayor parte de las investigaciones en este campo se seleccionan gliomas de bajo grado, este hecho se debe probablemente a su mejor pronóstico con respecto a los gliomas de alto grado.

En la misma línea, es escaso el número de estudios que analizan el rendimiento neuropsicológico de forma previa a la aplicación de los tratamientos oncológicos convencionales, es decir, previo a la cirugía y a las terapias adyuvantes (Scheibel y cols., 1996; Tucha, Smely, Preier y Lange, 2000; Reijneveld y cols., 2001; Alvarán, Gómez, Aguirre y Ortiz, 2008; 
Yoshii y cols., 2008). Es por ello que, en la actualidad no está determinado el papel del propio tumor en el daño neuropsicológico en esta población.

La mayoría de los estudios en esta área de conocimiento, han pretendido determinar el papel que desempeña la radioterapia en el funcionamiento neuropsicológico, años después de la aplicación de la misma (Taphoorn y cols., 1994; Vigliani y cols., 1994; Gregor y cols., 1996; Surma-aho y cols., 2001; Armstrong, Stern y Corn, 2001; Postma y cols., 2002; Klein y cols., 2002; Brown y cols., 2006; Torres y cols., 2003; Laack y cols., 2005; Douw y cols., 2009). Los hallazgos son contradictorios, ya que algunos autores han señalado que esta técnica no es la principal causa de deterioro neuropsicológico, informando que el tumor o la progresión del mismo y otros tratamientos sintomáticos (antiepileptógenos) pueden provocar dicho deterioro (Douw y cols., 2009; Laack y cols., 2005; Klein y cols., 2001; Tucha y cols., 2000).

La última limitación a considerar, hace referencia a la falta de acuerdo en la elección de las variables a estudiar y a la heterogeneidad de los instrumentos de valoración aplicados. Cabe señalar, que la mayoría de investigaciones incluyen variables de atención, memoria, función psicomotriz y lenguaje, medidas a partir de pruebas estandarizadas (Álvarez y Conde, 2009). En otros estudios, se analiza de forma exclusiva un único dominio neuropsicológico (Armstrong, Stern y Corn, 2001), y en otros, se valora el estado mental general mediante pruebas de screening (Brown y cols., 2003; Yoshii y cols., 2008). Como dato a resaltar, en los últimos años, se está incluyendo el estudio de componentes de las funciones ejecutivas, más allá de la atención alternante (Laack y cols., 2005; Douw y cols., 2009).

El presente trabajo tiene como objetivo definir el papel del tumor en el rendimiento neuropsicológico de pacientes afectados de glioma, desde el momento inicial de la enfermedad, intentando soslayar algunas de las limitaciones metodológicas anteriormente citadas. Para ello, se ha realizado una valoración de los aspectos neuropsicológicos implicados en la calidad de vida, en pacientes con sospecha radiológica de tumor cerebral primario, que van a ser sometidos a resección quirúrgica tumoral.

\section{Método}

\section{Participantes}

La muestra está constituida por 29 pacientes con sospecha radiológica de tumor cerebral primario, pertenecientes al Servicio de Neurocirugía del Hospital Clínico San Carlos de Madrid, que iban a ser sometidos a un procedimiento quirúrgico en el plazo de una semana posterior a la valoración neuropsicológica.

Para el estudio se aplicaron los siguientes criterios de inclusión: ser mayor de 18 años y menor de 75 , presentar sospecha radiológica de glioma y ser candidato a tratamiento quirúrgico en el plazo de una semana. Los criterios de exclusión utilizados fueron: tener enfermedad neurodegenerativa, historia psicopatológica severa, enfermedad oncológica previa a la actual, así como, presentar afasia de producción y compresión.

\section{Diseño}

El estudio presenta un diseño transversal, con carácter descriptivo y comparativo. Las variables independientes son: diagnóstico anatomopatológico (gliomas de bajo grado -I / II- y alto grado -III / IV) y hemisferio afectado (derecho e izquierdo). El grupo de variables dependientes está formado por las puntuaciones obtenidas en las pruebas neuropsicológicas, resumidas en la Tabla 1. Se controla el efecto de la edad y estado emocional (ansiedad y depresión), de acuerdo a lo referido en la literatura al uso (Perea y Ladera, 1995; Taphoorn, 2003).

\section{Variables $e$ instrumentos}

Las variables consideradas para la realización de 
Tabla 1. Variables neuropsicológicas registradas

\author{
Orientación-Atención \\ Orientación espacio-temporal-personal \\ Span atencional \\ Velocidad de procesamiento \\ de la información \\ Atención selectiva \\ Atención alternante

\section{Aprendizaje-Memoria} \\ Aprendizaje verbal \\ Recuerdo verbal libre a corto plazo \\ Recuerdo verbal libre a largo plazo \\ Recuerdo verbal claves a corto plazo \\ Recuerdo verbal claves largo plazo \\ Reconocimiento verbal \\ Memoria visual a corto plazo
}

\author{
Gnosias \\ Organización perceptual \\ Reconocimiento de objetos \\ Organización visual de estímulos \\ Habilidades visoconstructivas \\ Lenguaje \\ Denominación confrontación \\ Fluidez verbal semántica \\ Fluidez verbal fonológica \\ Funciones ejecutivas \\ Memoria de trabajo \\ Control mental \\ Habilidad espontánea de planificación y habilidad para seguir estrategia \\ concreta impuesta
}

la presente investigación son las variables sociodemográficas, clínicas y psicológicas, que se exponen a continuación.

- Variables sociodemográficas: edad, género, estado civil, situación laboral, nivel educativo. La información sobre ellas se registró a través de la historia clínica.

- Variables médicas: historia médica previa, antecedentes psicopatológicos previos, tratamiento farmacológico actual, tipo de cirugía propuesta, hemisferio afectado y diagnóstico anatomopatológico. La información relativa a todas ellas se obtuvo en la entrevista, historia clínica, resultados de pruebas de neuroimagen y estudio anatomopatológico.

- Variables psicológicas, se agruparon en reacciones emocionales y rendimiento en pruebas neuropsicológicas. La recogida de los datos de estas variables se realizó mediante la aplicación de un protocolo de valoración estandarizado, que aparece resumido en la Tabla 2.

\section{Procedimiento}

Esta investigación se realizó desde el 2008 al 2010, periodo en el cual se ha recogido la información de las variables neuropsicológicas de los participantes. Para su realización se acometieron las siguientes fases:

- Fase 0: Presentación del proyecto a la Jefatura de Servicio de Neurocirugía del Hospital Clínico San Carlos de Madrid y obtención del visto bueno de la Comisión de Docencia para la realización del estudio.

- Fase I: Presentación en las sesiones clínicas del Servicio de Neurocirugía de los pacientes con posible diagnóstico de tumor cerebral primario, que son susceptible de recibir tratamiento quirúrgico para resección tumoral.

- Fase II: Comprobación de criterios de inclusión y exclusión de cada candidato presentado.

- Fase III: Aplicación de las pruebas anteriormente citadas, siempre por la misma persona, en la misma ubicación intentado eliminar estímulos distractores, en turno de mañana para evitar el efecto de fatiga y decaimiento de procesos atencionales y en el mismo orden. La aplicación del protocolo constó de dos fases, que se dividen de la siguiente forma.

- Fase IIIa: Escala Hospitalaria Ansiedad y Depresión (HADS), Test de Aprendizaje Verbal España-Complutense (TAVEC), Subescala de span de dígitos directo-inverso de la WMS-III, Test de la campana, Trail Making Test (TMT), Test de organización visual de Hooper (HVOT) y Figuras incompletas WAIS.

- Fase IIIb: Figura compleja de Rey, Test de Denominación de Boston, Control Oral Word Test (FAS y animales), Subescala con- 
Tabla 2. Instrumentos de medida utilizados

\begin{tabular}{|c|c|c|c|}
\hline Instrumentos & Autores & Variable estudiada & Datos normativos españoles \\
\hline Memoria de Wechsler (WMS-III) & Wechsler (2004) & $\begin{array}{l}\text { Orientación espacio-temporal- } \\
\text { personal }\end{array}$ & $\begin{array}{l}\text { Puntuación escalar } \\
\text { Versión adaptada al castellano (Pereña y } \\
\text { cols., 2004) }\end{array}$ \\
\hline Memoria de Wechsler (WMS-III) & Wechsler (2004) & Span atencional & $\begin{array}{l}\text { Puntuación escalar } \\
\text { Versión adaptada al castellano (Pereña y } \\
\text { cols., 2004) }\end{array}$ \\
\hline $\begin{array}{l}\text { Trail Making Test B } \\
\text { TMT B }\end{array}$ & $\begin{array}{l}\text { Army Individual } \\
\text { Test Battery (1944) }\end{array}$ & Atención alternante & Puntuación centil errores \\
\hline $\begin{array}{l}\text { Trail Making Test A } \\
\text { TMT A }\end{array}$ & $\begin{array}{l}\text { Army Individual Test } \\
\text { Battery (1944) }\end{array}$ & $\begin{array}{l}\text { Velocidad de procesamiento } \\
\text { de la información }\end{array}$ & $\begin{array}{l}\text { Puntuación centil errores } \\
\text { Spreen y Strauss (1998) }\end{array}$ \\
\hline Test de la Campana & $\begin{array}{l}\text { Gauthier, Dehaut } \\
\text { y Joanette (1989) }\end{array}$ & Atención selectiva & $\begin{array}{l}\text { Punto corte } \\
\text { No existen estudios población española }\end{array}$ \\
\hline $\begin{array}{l}\text { Test de Aprendizaje Verbal España- } \\
\text { Complutense (TAVEC) }\end{array}$ & Benedet y Alexandre (1998) & $\begin{array}{l}\text { Aprendizaje verbal } \\
\text { Recuerdo verbal libre a corto plazo } \\
\text { Recuerdo verbal libre a largo plazo } \\
\text { Recuerdo verbal claves a corto plazo } \\
\text { Recuerdo verbal claves largo plazo }\end{array}$ & $\begin{array}{l}\text { Puntuación centil en cada escala } \\
\text { Muestra original española }\end{array}$ \\
\hline $\begin{array}{l}\text { Test de Copia y de reproducción } \\
\text { de memoria de figuras geométricas }\end{array}$ & $\begin{array}{l}\text { De la Cruz, Seisdedos y } \\
\text { Cordero (1997) }\end{array}$ & $\begin{array}{l}\text { Habilidades viso-constructivas } \\
\text { Memoria visual a corto plazo } \\
\text { complejas }\end{array}$ & $\begin{array}{l}\text { Puntuación centil } \\
\text { 6a Edición adaptación al castellano (1997) } \\
\text { TEA }\end{array}$ \\
\hline $\begin{array}{l}\text { Test de organización visual de } \\
\text { Hooper (HVOT) }\end{array}$ & Hooper (1983) & $\begin{array}{l}\text { Organización perceptual } \\
\text { Reconocimiento de objetos }\end{array}$ & $\begin{array}{l}\text { Punto corte } \\
\text { No existen datos publicados en población } \\
\text { española }\end{array}$ \\
\hline Figuras incompletas WAIS III & Wechsler (1999) & Organización visual de estímulos & $\begin{array}{l}\text { Puntuación escalar } \\
\text { Adaptación al castellano TEA }\end{array}$ \\
\hline $\begin{array}{l}\text { Control Oral Word Test (COWAT) } \\
\text { FAS } \\
\text { Animales }\end{array}$ & Benton y Hamsher (1989) & $\begin{array}{l}\text { Fluidez verbal fonológica } \\
\text { Fluidez verbal semántica }\end{array}$ & $\begin{array}{l}\text { Puntuación centil } \\
\text { Spreen y Strauss (1998) }\end{array}$ \\
\hline Test de Denominación de Boston & $\begin{array}{l}\text { Kaplan, Goodglass } \\
\text { y Weintraub (1983) }\end{array}$ & Denominación confrontación & $\begin{array}{l}\text { Puntuación centil } \\
\text { Spreen y Strauss (1998) }\end{array}$ \\
\hline $\begin{array}{l}\text { Escala Letras y Números } \\
\text { Test Memoria de Wechsler (WMS-III) }\end{array}$ & Wechsler (2004) & Memoria de trabajo & $\begin{array}{l}\text { Puntuación escalar } \\
\text { Versión adaptada al castellano (Pereña y } \\
\text { cols., 2004) }\end{array}$ \\
\hline $\begin{array}{l}\text { Escala Control Mental } \\
\text { Test Memoria de Wechsler (WMS-III) }\end{array}$ & Wechsler (2004) & Control mental & $\begin{array}{l}\text { Puntuación escalar } \\
\text { Versión adaptada al castellano (Pereña y } \\
\text { cols., 2004) }\end{array}$ \\
\hline $\begin{array}{l}\text { Mapa Zoo } \\
\text { (Escala de BADS: Behaviorual } \\
\text { assessment of the dysexecutive } \\
\text { syndrome) }\end{array}$ & $\begin{array}{l}\text { Alderman, Burgess, } \\
\text { Emslie, Evans } \\
\text { y Wilson (1996) }\end{array}$ & $\begin{array}{l}\text { Habilidad espontánea de planificación } \\
\text { y habilidad para seguir } \\
\text { estrategia concreta impuesta }\end{array}$ & $\begin{array}{l}\text { Puntuación perfil } \\
\text { No existen datos publicados en población } \\
\text { española }\end{array}$ \\
\hline $\begin{array}{l}\text { Escala Hospitalaria Ansiedad } \\
\text { y Depresión HADS }\end{array}$ & Zigmond y Snaith (1983) & $\begin{array}{l}\text { Malestar psicológico, nivel ansiedad } \\
\text { y depresión }\end{array}$ & $\begin{array}{l}\text { Punto de corte } \\
\text { Adaptación población española }\end{array}$ \\
\hline
\end{tabular}

trol mental de la WMS-III, subescala de letras y números de de la WMS-III y Subtest del Mapa de Zoo de Behaviorual assessment of the dysexecutive síndrome (BADS).

- Fase IV: análisis estadísticos y obtención de resultados.

\section{Análisis estadísticos}

Estos se han realizado con el paquete estadístico SPSS 15.0. Para el primer objetivo de la investigación (descripción sociodemográfica y clínica de la muestra seleccionada) se ha realizado un análisis 
descriptivo y de frecuencias para las variables sociodemográficas, clínicas y psicológicas, anteriormente citadas.

El segundo objetivo pretende ver si existen diferencias en el rendimiento neuropsicológico en función de la anatomía patológica y del hemisferio afecto. Pero dado que existen variables tales como la edad, la ansiedad y la depresión que pueden influir en dicho rendimiento, se hace necesario comprobar en primer lugar si efectivamente existen estas diferencias ( $t$ de Student para muestras independientes) $y$, en el caso de su existencia, controlar el efecto de las mismas (análisis de covarianza).

\section{Resultados}

\section{A) Resultados descriptivos sociodemográficos y clínicos}

De la muestra total, el $62 \%$ fueron hombres y el $38 \%$ restante fueron mujeres, la edad media fue de 54 años (D.T.: 14.1), en un rango de 24 a 72 años. En relación al nivel educativo, el 44,8\% no tenían estudios o no había finalizado la educación primaria, el $20,7 \%$ tenían estudios primarios finalizados y el mismo porcentaje estudios secundarios, sólo el 13,8\% de la muestra valorada poseía estudios superiores.

En relación a las variables médicas, el 69\% de la muestra presentó diagnóstico anatomopatológico de glioma del alto grado (astrocitoma anaplásico y glioblastoma multiforme). Los síntomas clínicos iniciales más frecuentes fueron cefalea y alteraciones motoras, seguidos de síntomas emocionales y déficits del lenguaje. (Véase Gráfico 1).

Respecto a la localización de las lesiones, el 59\% de la muestra presentó afectación del hemisferio derecho, mientras que únicamente el $7 \%$ presento lesión bilateral. En la distribución de acuerdo al lóbulo afectado, se observa que más del $40 \%$ de la muestra presentaba afectación del lóbulo frontal (Véase Tabla 3).

En relación a las variables neuropsicológicas, en este apartado se presentan las puntuaciones centiles, ajustadas a su grupo de edad según los manuales de correcciones de cada prueba, para obtener informa-

Gráfico 1. Síntomas clínicos en el momento diagnóstico

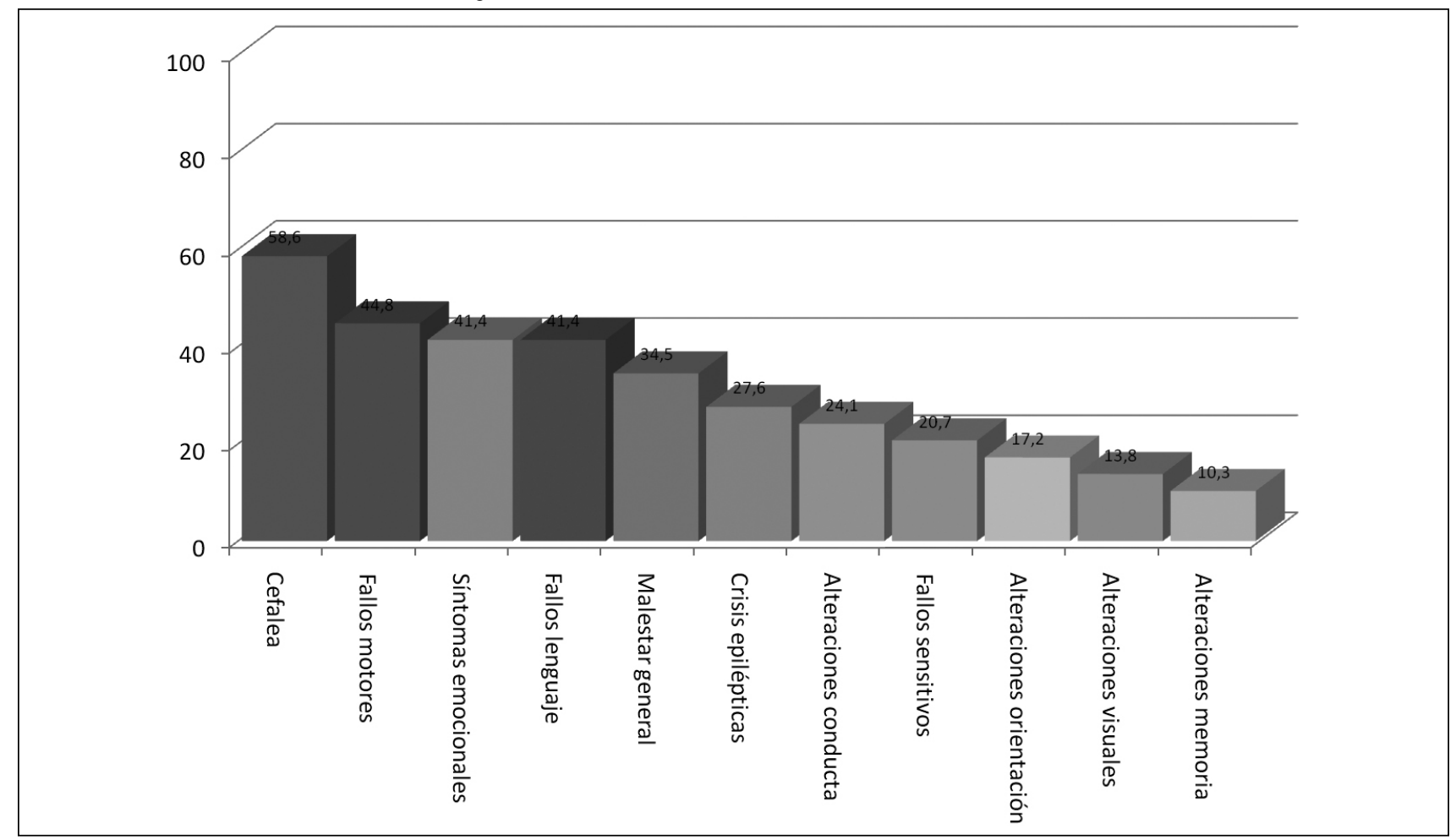


Tabla 3. Localización por hemisferio

\begin{tabular}{lc}
\hline & Porcentaje \\
\hline Frontal & $48 \%$ \\
Temporal & $7 \%$ \\
Parietal & $27 \%$ \\
Occipital & $10 \%$ \\
Más de un lóbulo afectado & $7 \%$ \\
\hline
\end{tabular}

ción clínica de toda la muestra. Así, se puede observar que más del $50 \%$ de la muestra total presenta puntuaciones por debajo del centil 40 o por debajo del punto de corte establecido por la prueba, excepto en la escala de orientación de la escala WMS-III y la tarea de recuerdo verbal con claves a corto plazo del TAVEC (ver Gráfico 2). De forma específica, más del $80 \%$ obtiene puntuaciones centiles por debajo de 40 en TMT-A y en la tarea de memoria inmediata de la Figura Compleja Rey, lo que implica fallos en velocidad procesamiento información y recuerdo visual inmediato. En el Test de Denominación de Boston, se observa que el $82 \%$ de la muestra presenta puntuaciones centiles menores del 25 , lo que indica problemas severos en nominación por confrontación visual.

\section{B) Diferencias de las variables hemisferio afecta- do y diagnostico anatomopatológico de acuerdo a la edad y el malestar emocional}

\section{b.1) Edad}

De acuerdo a la literatura actual, es necesario considerar el efecto de la variable edad y del estado emocional en el rendimiento neuropsicológico de los pacientes (Perea y Ladera, 1995; Taphoorn, 2003). En este estudio se registro en la entrevista inicial la edad de cada paciente y el estado emocional mediante la Escala de Ansiedad y Depresión Hospitalaria (HADS; Zigmond y Snaith, 1983). Para identificar si existían diferencias significativas en

Gráfico 2. Porcentajes bajo rendimiento pruebas neuropsicológicas

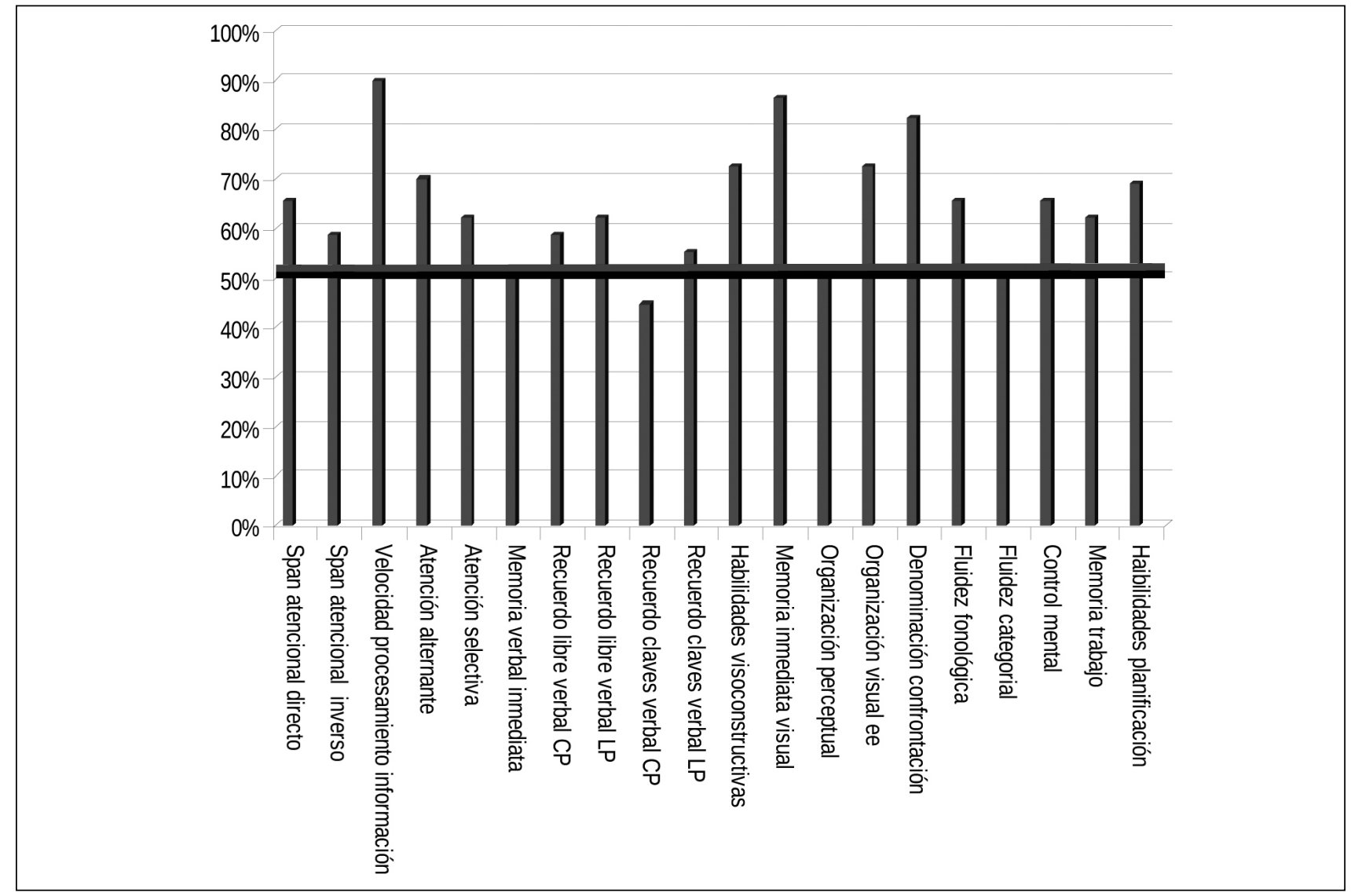


estas variables de acuerdo al diagnóstico histopatológico y al hemisferio afectado, se ha utilizado la $t$ de Student para muestras independientes.

La edad media para los pacientes con diagnóstico anatomopatológico de glioma de alto grado (III-IV) fue de 60.75 (S.D.: 9.99) y para el grupo de glioma de bajo grado (I-II) de 41.44 años (S.D.: 12.79). Así, se observaron diferencias estadísticamente significativas para la variable edad entre los dos grupos $t$ $(27)=4.41 ; \mathrm{p}=.000(\mathrm{p}<.001)$. Por el contrario, no se observaron diferencias en la edad de los grupos de acuerdo al hemisferio afectado $\mathrm{t}(25)=.15 ; \mathrm{p}=$ .88 , de tal forma que los pacientes que tenían afectado el hemisferio izquierdo tenían una edad media de 56.10 (S.D.: 12.43) y los que presentaban el tumor en el hemisferio derecho fue de 55.24 (S.D.: 15.53).

\section{b.2) Malestar emocional}

La puntuación obtenida en las variables emociona- les de ansiedad y depresión (escala HADS) para los pacientes con diagnóstico anatomopatológico de glioma de alto grado (III-VI) fue de 13.65 (S.D.: 7.86) y para el grupo de glioma de bajo grado (I-II) fue de 13.33 (S.D.: 7.73); no alcanzaron la significación estadísticas estas diferencias $\mathrm{t}(27)=1.01 ; \mathrm{p}=.92$. Tampoco, se observaron dichas diferencias respecto al hemisferio afectado $\mathrm{t}(25)=-.39 ; \mathrm{p}=.69$. Los pacientes que tenían afectado el hemisferio izquierdo obtuvieron una puntuación media en esta escala de 12.40 (S.D.: 7.54) y los que presentaban el tumor en el hemisferio derecho fue de 13.65 (S.D.: 8.26).

\section{C) Diferencias de las variables neuropsicológicas en función a las variables independientes}

\section{c.1) Diferencias de las variables neuropsicológicas en función del diagnóstico anatomopatológico}

En la Tabla 4 se exponen las puntuaciones obtenidas en cada grupo para las variables neuropsicológi-

Tabla 4. Puntuaciones medias de las variables neuropsicológicas de acuerdo al diagnóstico anatomopatológico

\begin{tabular}{|c|c|c|c|c|}
\hline \multirow[b]{2}{*}{ Variables } & \multicolumn{2}{|c|}{ Glioma bajo grado } & \multicolumn{2}{|c|}{ Glioma alto grado } \\
\hline & Media & $D . T$ & Media & $D . T$ \\
\hline Orientación & 12.44 & .527 & 11.45 & 1.66 \\
\hline Span atención directo & 8.00 & 2.64 & 6.70 & 1.8 \\
\hline Span atención inverso & 5.44 & 2.40 & 4.05 & 1.57 \\
\hline Atención selectiva & 30.67 & 6.42 & 24.85 & 8.35 \\
\hline Velocidad procesamiento información & 44.33 & 20.60 & 115.30 & 95.58 \\
\hline Atención alternante & 153 & 155.05 & 257.25 & 101.02 \\
\hline Aprendizaje verbal $1^{\mathrm{er}}$ ensayo & 6.33 & 1.80 & 5.3 & 2.15 \\
\hline Aprendizaje verbal $5^{\circ}$ ensayo & 13.44 & 2.45 & 9.7 & 3.71 \\
\hline Aprendizaje verbal total & 55.77 & 11.82 & 39.75 & 14.54 \\
\hline Interferencia & 4.22 & 1.56 & 5.05 & 1.93 \\
\hline Recuerdo verbal libre $\mathrm{CP}$ & 11.0 & 3.80 & 6.9 & 5.07 \\
\hline Recuerdo verbal libre LP & 10.33 & 4.27 & 7.5 & 5.06 \\
\hline Recuerdo verbal clave $\mathrm{CP}$ & 11.66 & 3.87 & 8.0 & 4.94 \\
\hline Recuerdo verbal clave LP & 11.88 & 3.85 & 7.6 & 4.80 \\
\hline Reconocimiento verbal & 14.33 & 2.17 & 12.75 & 3.30 \\
\hline Organización perceptual & 15.00 & 5.36 & 8.10 & 5.24 \\
\hline Organización visual ee & 23.89 & 4.37 & 15.25 & 6.74 \\
\hline Habilidades visoconstructivas & 28.55 & 7.71 & 18.07 & 10.91 \\
\hline Memoria inmediata visual & 17.66 & 8.73 & 7.45 & 7.23 \\
\hline Memoria demorada visual & 16.05 & 7.41 & 7.02 & 6.50 \\
\hline Denominación verbal confrontación & 51.56 & 6.16 & 39.3 & 10.92 \\
\hline Fluidez fonológica & 37.33 & 15.14 & 25.65 & 11.33 \\
\hline Fluidez categorial & 20.67 & 9.92 & 15.7 & 6.20 \\
\hline Control mental & 25.56 & 7.36 & 16.9 & 6.82 \\
\hline Memoria trabajo & 10.00 & 3.74 & 4.7 & 4.14 \\
\hline Habilidades espontanea planificación y habilidad para seguir estrategia concreta impuesta & 14.55 & 2.96 & 2.35 & 6.36 \\
\hline
\end{tabular}


cas, observándose un mejor rendimiento del grupo de pacientes con diagnóstico anatomopatológico de glioma de bajo grado (I-II), con respecto a los pacientes con gliomas de alto grado (III-IV).

Dado que la edad influye en el rendimiento neuropsicológico, se intento comparar este rendimiento entre gliomas de alto y bajo grado, controlando el efecto de la edad. Para ello, se realizaron ANCOVAS utilizando como variable independiente el diagnóstico anatomopatológico, como covariante la variable edad y como variable dependiente todas las variables neuropsicológicas. De dicho análisis, se deriva que la anatomía patológica explica las diferencias únicamente en el dominio de habilidades de planificación y para seguir estrategia concreta impuesta (Mapa del Zoo del BADS) $(\mathrm{F}(1 / 26)=$ 8.72; $\mathrm{MCe}=26.492 ; \mathrm{p}=.007)$; donde se identificó que los pacientes con gliomas de bajo grado presentan un mejor rendimiento para esta variable, con respecto a los gliomas de alto grado. No se alcanzó la significación estadística para el resto de variables neuropsicológicas en función de dicho diagnóstico anatomopatológico.

Por el contrario, la edad es capaz de explicar las diferencias en el rendimiento neuropsicológico entre gliomas de alto y bajo grado en las siguientes variables neuropsicológicas. Así, en dominios de atención se observo efecto de la variable edad en span atencional indirecta $(\mathrm{F}(1 / 26)=10.125$; MCerror $=2.57$; $\mathrm{p}=.004$; en el TMT-A (velocidad de procesamiento información $)(\mathrm{F}(1 / 26)=5.39 ; \mathrm{MCe}=5637.215 ; \mathrm{p}=$ .028) y en el TMT-B (atención alternante) $(\mathrm{F}(1 / 26)=$ 10.037; $\mathrm{MCe}=10781.429 ; \mathrm{p}=.004)($ tabla 5$)$.

En memoria verbal valorado mediante la escala TAVEC, se identificó un efecto significativo de la variable edad en el aprendizaje verbal $5^{\circ}$ ensayo y en aprendizaje verbal total $(\mathrm{F}(1 / 26)=6.716 ; \mathrm{MCe}=$ 9.488; $\mathrm{p}=.0015 ; \mathrm{F}(1 / 26)=6.319 ; \mathrm{MCe}=159.078$; $\mathrm{p}=.018$, respectivamente); así como, en el recuerdo verbal libre a corto plazo $(\mathrm{F}(1 / 26)=7.260 ; \mathrm{MCe}=$ $18.214 ; \mathrm{p}=.012)$ y recuerdo con claves a corto plazo $(\mathrm{F}(1 / 26)=6.616 ;$ MCerror $=17.905 ; \mathrm{p}=.016)$ (tabla 6).

En la misma línea, dicho efecto se evidenció también en tareas de memoria visual, de forma específica en habilidades visocontructivas $(\mathrm{F}(1 / 26)=$ 16.423; $\mathrm{MCe}=64.596 ; \mathrm{p}=.021)$, recuerdo visual inmediato y demorado, recogido mediante la Figura

Tabla 5. Resultados ANCOVA. Edad x diagnóstico anatomopatológico para las medidas de orientación y atención

\begin{tabular}{|c|c|c|c|}
\hline \multicolumn{4}{|l|}{ Orientación } \\
\hline Edad & $F(1 / 26)=.988$ & $\mathrm{MC}_{\mathrm{error}}=2.044$ & $\mathrm{p}=.329$ \\
\hline Diagnóstico anatomopatológico & $\mathrm{F}(1 / 26)=.459$ & $\mathrm{MC}_{\text {error }}=2.044$ & $\mathrm{p}=.504$ \\
\hline \multicolumn{4}{|l|}{ Span atención directo } \\
\hline Edad & $F(1 / 26)=2.75$ & $\mathrm{MC}_{\text {error }}=4.11$ & $p=.109$ \\
\hline Diagnóstico anatomopatológico & $\mathrm{F}(1 / 26)=.021$ & $\mathrm{MC}_{\text {error }}=4.11$ & $\mathrm{p}=.889$ \\
\hline \multicolumn{4}{|l|}{ Span atención inverso } \\
\hline Edad & $F(1 / 26)=10.125$ & $\mathrm{MC}_{\text {error }}=2.57$ & $\mathrm{p}=.004^{*}$ \\
\hline Diagnóstico anatomopatológico & $F(1 / 26)=.168$ & $\mathrm{MC}_{\text {error }}=2.57$ & $\mathrm{p}=.685$ \\
\hline \multicolumn{4}{|l|}{ Atención selectiva } \\
\hline$\overline{\text { Edad }}$ & $F(1 / 26)=3.016$ & $\mathrm{MC}_{\text {error }}=57.091$ & $p=.094$ \\
\hline Diagnóstico anatomopatológico & $\mathrm{F}(1 / 26)=.114$ & $\mathrm{MC}_{\mathrm{error}}=57.091$ & $\mathrm{p}=.738$ \\
\hline \multicolumn{4}{|c|}{ Velocidad procesamiento información } \\
\hline$\overline{\text { Edad }}$ & $F(1 / 26)=5.39$ & $\mathrm{MC}_{\text {error }}=5637.21$ & $\mathrm{p}=.028 *$ \\
\hline Diagnóstico anatomopatológico & $F(1 / 26)=.85$ & $\mathrm{MC}_{\text {error }}=5637.21$ & $\mathrm{p}=.772$ \\
\hline \multicolumn{4}{|l|}{ Atención alternante } \\
\hline Edad & $F(1 / 26)=10.037$ & $\mathrm{MC}_{\text {error }}=10718.42$ & $\mathrm{p}=.004 * *$ \\
\hline Diagnóstico anatomopatológico & $F(1 / 26)=.019$ & $\mathrm{MC}_{\mathrm{error}}=10718.42$ & $\mathrm{p}=.891$ \\
\hline
\end{tabular}

${ }^{*} \mathrm{p}<.05 ; * * \mathrm{p}<.01 ; * * * \mathrm{p}<.001$ 
Tabla 6. Resultados ANCOVA. Edad x diagnóstico anatomopatológico para las medidas de memoria verbal

\begin{tabular}{|c|c|c|c|}
\hline \multicolumn{4}{|l|}{ Aprendizaje verbal $1^{\mathrm{er}}$ ensayo } \\
\hline $\begin{array}{l}\text { Edad } \\
\text { Diagnóstico anatomopatológico }\end{array}$ & $\begin{array}{l}\mathrm{F}(1 / 26)=3.108 \\
\mathrm{~F}(1 / 26)=.023\end{array}$ & $\begin{array}{l}\mathrm{MC}_{\text {error }}=3.923 \\
\mathrm{MC}_{\text {error }}=3.923\end{array}$ & $\begin{array}{l}\mathrm{p}=.090 \\
\mathrm{p}=.882\end{array}$ \\
\hline \multicolumn{4}{|l|}{ Aprendizaje verbal $5^{\circ}$ ensayo } \\
\hline $\begin{array}{l}\text { Edad } \\
\text { Diagnóstico anatomopatológico }\end{array}$ & $\begin{array}{l}F(1 / 26)=6.71 \\
F(1 / 26)=.399\end{array}$ & $\begin{array}{l}\mathrm{MC}_{\text {error }}=246.697 \\
\mathrm{MC}_{\text {error }}=246.697\end{array}$ & $\begin{array}{l}\mathrm{p}=.015^{*} \\
\mathrm{p}=.533\end{array}$ \\
\hline \multicolumn{4}{|l|}{ Aprendizaje verbal total } \\
\hline $\begin{array}{l}\text { Edad } \\
\text { Diagnóstico anatomopatológico }\end{array}$ & $\begin{array}{l}F(1 / 26)=6.319 \\
F(1 / 26)=.619\end{array}$ & $\begin{array}{l}\mathrm{MC}_{\text {error }}=4136.028 \\
\mathrm{MC}_{\text {error }}=4136.028\end{array}$ & $\begin{array}{l}\mathrm{p}=.018^{*} \\
\mathrm{p}=.439\end{array}$ \\
\hline \multicolumn{4}{|l|}{ Interferencia } \\
\hline $\begin{array}{l}\text { Edad } \\
\text { Diagnóstico anatomopatológico }\end{array}$ & $\begin{array}{l}F(1 / 26)=.000 \\
F(1 / 26)=.717\end{array}$ & $\begin{array}{l}\mathrm{MC}_{\text {error }}=3.481 \\
\mathrm{MC}_{\text {error }}=3.481\end{array}$ & $\begin{array}{l}\mathrm{p}=.995 \\
\mathrm{p}=.405\end{array}$ \\
\hline \multicolumn{4}{|l|}{ Recuerdo verbal libre CP } \\
\hline $\begin{array}{l}\text { Edad } \\
\text { Diagnóstico anatomopatológico }\end{array}$ & $\begin{array}{l}F(1 / 26)=7.26 \\
F(1 / 26)=.119\end{array}$ & $\begin{array}{l}\mathrm{MC}_{\mathrm{error}}=18.214 \\
\mathrm{MC}_{\text {error }}=18.214\end{array}$ & $\begin{array}{l}\mathrm{p}=.012 * \\
\mathrm{p}=.936\end{array}$ \\
\hline \multicolumn{4}{|l|}{ Recuerdo verbal libre LP } \\
\hline $\begin{array}{l}\text { Edad } \\
\text { Diagnóstico anatomopatológico }\end{array}$ & $\begin{array}{l}F(1 / 26)=2.66 \\
F(1 / 26)=.008\end{array}$ & $\begin{array}{l}\mathrm{MC}_{\mathrm{error}}=22.081 \\
\mathrm{MC}_{\text {error }}=22.081\end{array}$ & $\begin{array}{l}\mathrm{p}=.114 \\
\mathrm{p}=.930\end{array}$ \\
\hline \multicolumn{4}{|l|}{ Recuerdo verbal clave CP } \\
\hline $\begin{array}{l}\text { Edad } \\
\text { Diagnóstico anatomopatológico }\end{array}$ & $\begin{array}{l}F(1 / 26)=6.616 \\
F(1 / 26)=.000\end{array}$ & $\begin{array}{l}\mathrm{MC}_{\mathrm{error}}=17.905 \\
\mathrm{MC}_{\text {error }}=17.905\end{array}$ & $\begin{array}{l}\mathrm{p}=.016^{*} \\
\mathrm{p}=.985\end{array}$ \\
\hline \multicolumn{4}{|l|}{ Recuerdo verbal clave $\mathbf{L P}$} \\
\hline $\begin{array}{l}\text { Edad } \\
\text { Diagnóstico anatomopatológico }\end{array}$ & $\begin{array}{l}F(1 / 26)=4.136 \\
F(1 / 26)=.334\end{array}$ & $\begin{array}{l}\mathrm{MC}_{\text {error }}=18.507 \\
\mathrm{MC}_{\text {error }}=18.507\end{array}$ & $\begin{array}{l}\mathrm{p}=.052 \\
\mathrm{p}=.568\end{array}$ \\
\hline \multicolumn{4}{|l|}{ Reconocimiento verbal } \\
\hline $\begin{array}{l}\text { Edad } \\
\text { Diagnóstico anatomopatológico }\end{array}$ & $\begin{array}{l}F(1 / 26)=.292 \\
F(1 / 26)=.402\end{array}$ & $\begin{array}{l}\mathrm{MC}_{\text {error }}=9.347 \\
\mathrm{MC}_{\text {error }}=9.347\end{array}$ & $\begin{array}{l}\mathrm{p}=.594 \\
\mathrm{p}=.532\end{array}$ \\
\hline
\end{tabular}

$* \mathrm{p}<.05 ; * * \mathrm{p}<.01 ; * * * \mathrm{p}<.001$

Compleja Rey $(\mathrm{F}(1 / 26)=36.158 ; \mathrm{MCe}=25.829 ; \mathrm{p}$ $=.000 ; \mathrm{F}(1 / 26)=35.86 ; \mathrm{MCe}=20.124 ; \mathrm{p}=.000$, respectivamente) (tabla 7 ).

Por otra parte, en las dos medidas de organización visual valorada con HVOP y Figuras incompletas del WAIS $(\mathrm{F}(1 / 26)=6.780 ; \mathrm{MCe}=22.935 ; \mathrm{p}=$ $.015 ; \mathrm{F}(1 / 26)=25.432 ; \mathrm{MCe}=19.767 ; \mathrm{p}=.000$, respectivamente) (tabla 8$)$.

Tabla 7. Resultados ANCOVA. Edad x diagnóstico anatomopatológico para las medidas de memoria visual

\section{Habilidades visoconstructivas}

\begin{tabular}{|c|c|c|c|}
\hline $\begin{array}{l}\text { Edad } \\
\text { Diagnóstico anatomopatológico }\end{array}$ & $\begin{array}{l}\mathrm{F}(1 / 26)=16.423 \\
\mathrm{~F}(1 / 26)=.021\end{array}$ & $\begin{array}{l}\mathrm{MC}_{\text {error }}=64.596 \\
\mathrm{MC}_{\text {error }}=64.596\end{array}$ & $\begin{array}{l}\mathrm{p}=.000 * * * \\
\mathrm{p}=.88\end{array}$ \\
\hline \multicolumn{4}{|l|}{ Memoria inmediata visual } \\
\hline $\begin{array}{l}\text { Edad } \\
\text { Diagnóstico anatomopatológico }\end{array}$ & $\begin{array}{l}F(1 / 26)=36.15 \\
F(1 / 26)=0.005\end{array}$ & $\begin{array}{l}\mathrm{MC}_{\text {error }}=25.829 \\
\mathrm{MC}_{\text {error }}=25.829\end{array}$ & $\begin{array}{l}\mathrm{p}=.000 * * * \\
\mathrm{p}=.942\end{array}$ \\
\hline \multicolumn{4}{|l|}{ Memoria demorada visual } \\
\hline $\begin{array}{l}\text { Edad } \\
\text { Diagnóstico anatomopatológico }\end{array}$ & $\begin{array}{l}F(1 / 26)=35.865 \\
F(1 / 26)=.003\end{array}$ & $\begin{array}{l}\mathrm{MC}_{\text {error }}=20.124 \\
\mathrm{MC}_{\text {error }}=20.124\end{array}$ & $\begin{array}{l}\mathrm{p}=.000 * * * \\
\mathrm{p}=.959\end{array}$ \\
\hline
\end{tabular}

${ }^{*} \mathrm{p}<.05 ; * * \mathrm{p}<.01 ; * * * \mathrm{p}<.001$ 
Tabla 8. Resultados ANCOVA. Edad x diagnóstico anatomopatológico para las medidas de organización perceptiva

\begin{tabular}{llll}
\hline Organización perceptual & & & \\
\hline Edad & $\mathrm{F}(1 / 26)=25.432$ & $\mathrm{MC}_{\text {error }}=19.767$ & $\mathrm{p}=.000^{* * *}$ \\
Diagnóstico anatomopatológico & $\mathrm{F}(1 / 26)=.182$ & $\mathrm{MC}_{\text {error }}=19.767$ & $\mathrm{p}=.673$ \\
\hline Organización visual ee & & & $\mathrm{p}=.015^{*}$ \\
\hline Edad & $\mathrm{F}(1 / 26)=6.78$ & $\mathrm{MC}_{\text {error }}=22.935$ & $\mathrm{p}=.303$ \\
Diagnóstico anatomopatológico & $\mathrm{F}(1 / 26)=1.105$ & $\mathrm{MC}_{\text {error }}=22.935$ & \\
\hline
\end{tabular}

${ }^{*} \mathrm{p}<.05 ; * * \mathrm{p}<.01 ; * * * \mathrm{p}<.001$

Tabla 9. Resultados ANCOVA. Edad x diagnóstico anatomopatológico para las medidas de lenguaje

\begin{tabular}{llll}
\hline Denominación verbal confrontación & & & \\
\hline Edad & $\mathrm{F}(1 / 26)=7.57$ & $\mathrm{MC}_{\text {error }}=59.66$ & $\mathrm{p}=.011^{*}$ \\
Diagnóstico anatomopatológico & $\mathrm{F}(1 / 26)=.255$ & $\mathrm{MC}_{\text {error }}=59.66$ & $\mathrm{p}=.618$ \\
\hline Fluidez fonológica & & & $\mathrm{p}=.019^{*}$ \\
\hline Edad & $\mathrm{F}(1 / 26)=6.292$ & $\mathrm{MC}_{\text {error }}=132.372$ & $\mathrm{p}=.763$ \\
Diagnóstico anatomopatológico & $\mathrm{F}(1 / 26)=.093$ & $\mathrm{MC}_{\text {error }}=132.372$ & \\
\hline Fluidez categorial & & & $\mathrm{p}=.017^{*}$ \\
\hline Edad & $\mathrm{F}(1 / 26)=6.471$ & $\mathrm{MC}_{\text {error }}=46.817$ & $\mathrm{p}=.791$ \\
Diagnóstico anatomopatológico & $\mathrm{F}(1 / 26)=.072$ & $\mathrm{MC}_{\text {error }}=46.817$ & \\
\hline
\end{tabular}

$* \mathrm{p}<.05 ; * * \mathrm{p}<.01 ; * * * \mathrm{p}<.001$

Tabla 10. Resultados ANCOVA. Edad x diagnóstico anatomopatológico para las medidas de funciones ejecutivas

\begin{tabular}{llll}
\hline Control mental & & & \\
\hline Edad & $\mathrm{F}(1 / 26)=10.518$ & $\mathrm{MC}_{\text {error }}=36.147$ & $\mathrm{p}=.003^{* *}$ \\
Diagnóstico anatomopatológico & $\mathrm{F}(1 / 26)=.404$ & $\mathrm{MC}_{\text {error }}=36.147$ & $\mathrm{p}=.531$ \\
\hline Memoria trabajo & & & $\mathrm{p}=.000^{* * *}$ \\
\hline Edad & $\mathrm{F}(1 / 26)=20.22$ & $\mathrm{MC}_{\text {error }}=9.48$ & $\mathrm{p}=.723$ \\
Diagnóstico anatomopatológico & $\mathrm{F}(1 / 26)=.129$ & $\mathrm{MC}_{\text {error }}=9.48$ & $\mathrm{p}=.024^{*}$ \\
\hline Habilidades espontanea planificación y habilidad para seguir estrategia concreta impuesta & $\mathrm{p}=.007 * *$ \\
\hline Edad & $\mathrm{F}(1 / 26)=5.737$ & $\mathrm{MC}_{\text {error }}=26.492$ & $\mathrm{MC}_{\text {error }}=26.492$ \\
Diagnóstico anatomopatológico & $\mathrm{F}(1 / 26)=8.725$ & & \\
\hline
\end{tabular}

${ }^{*} \mathrm{p}<.05 ; * * \mathrm{p}<.01 ; * * * \mathrm{p}<.001$

Para las pruebas que valoraban lenguaje, se observo efecto de la variable edad en la puntuación del Test Denominación Boston (denominación verbal por confrontación $)(\mathrm{F}(1 / 26)=7.57$; $\mathrm{MCe}=59.66$; $\mathrm{p}$ $=.011)$, en el FAS que valora la fluidez fonológica $(\mathrm{F}(1 / 26)=6.292 ; \mathrm{MCe}=132.372 ; \mathrm{p}=.019)$ y en la fluidez de animales (fluidez categorial) $(\mathrm{F}(1 / 26)=$ 6.471; $\mathrm{MCe}=46.817 ; \mathrm{p}=.017$ ) (tabla 9).

En último lugar, se observo efecto de la variable edad en las siguientes medidas de funciones ejecutivas: Letras y números del WMS-III (memoria de trabajo $)(\mathrm{F}(1 / 26)=20.22 ; \mathrm{MCe}=9.48 ; \mathrm{p}=.000) \mathrm{y}$
Escala de control mental del WMS-III $(\mathrm{F}(1 / 26)=$ 10.518; $\mathrm{MCe}=36.147 ; \mathrm{p}=.003)($ tabla 10$)$.

\section{c.2) Diferencias de las variables neuropsicológicas en función al hemisferio afectado}

En la Tabla 11 se muestran las puntuaciones medias de las variables neuropsicológicas en función del hemisferio afectado.

Al no observarse diferencias en la variable edad y variables emocionales (ansiedad y depresión) entre 
Tabla 11. Puntuaciones medias de las variables neuropsicológicas de acuerdo al hemisferio afectado

\begin{tabular}{|c|c|c|c|c|}
\hline \multirow[b]{2}{*}{ Variables } & \multicolumn{2}{|c|}{ Hemisferio derecho } & \multicolumn{2}{|c|}{ Hemisferio izquierdo } \\
\hline & Media & $D . T$ & Media & $D . T$ \\
\hline Orientación & 11.65 & 1.49 & 12.00 & 1.49 \\
\hline Span atención directo & 7.06 & 2.41 & 7.20 & 1.98 \\
\hline Span atención inverso & 4.41 & 2.32 & 4.50 & 1.43 \\
\hline Atención selectiva & 27.06 & 6.32 & 25.70 & 11.35 \\
\hline Velocidad procesamiento información & 112.24 & 100.38 & 72.20 & 57.76 \\
\hline Atención alternante & 225.76 & 117.77 & 237.40 & 151.28 \\
\hline Aprendizaje verbal $1^{\mathrm{er}}$ ensayo & 5.23 & 1.92 & 5.50 & 1.77 \\
\hline Aprendizaje verbal $5^{\circ}$ ensayo & 11.0 & 3.87 & 10.20 & 3.96 \\
\hline Aprendizaje verbal total & 45.05 & 15.80 & 41.80 & 16.20 \\
\hline Interferencia & 4.82 & 1.77 & 4.30 & 1.88 \\
\hline Recuerdo verbal libre $\mathrm{CP}$ & 8.11 & 5.07 & 7.50 & 5.23 \\
\hline Recuerdo verbal libre LP & 8.17 & 4.65 & 8.30 & 5.90 \\
\hline Recuerdo verbal clave $\mathrm{CP}$ & 9.47 & 4.58 & 8.10 & 5.83 \\
\hline Recuerdo verbal clave LP & 9.41 & 4.50 & 7.70 & 5.94 \\
\hline Reconocimiento verbal & 13.29 & 2.51 & 13.00 & 4.18 \\
\hline Organización perceptual & 9.82 & 6.52 & 11.40 & 5.85 \\
\hline Organización visual ee & 17.12 & 8.21 & 18.20 & 5.90 \\
\hline Habilidades visoconstructivas & 18.61 & 12.24 & 24.40 & 8.61 \\
\hline Memoria inmediata visual & 10.00 & 10.18 & 10.65 & 7.55 \\
\hline Memoria demorada visual & 8.97 & 9.10 & 10.35 & 6.27 \\
\hline Denominación verbal confrontación & 42.82 & 11.13 & 42.00 & 12.35 \\
\hline Fluidez fonológica & 29.65 & 15.17 & 27.50 & 11.95 \\
\hline Fluidez categorial & 16.82 & 7.78 & 16.60 & 8.07 \\
\hline Control mental & 18.94 & 8.37 & 20.50 & 8.07 \\
\hline Memoria trabajo & 10.00 & 3.74 & 6.90 & 3.92 \\
\hline Habilidades espontánea planificación y habilidad para seguir estrategia concreta impuesta & 5.52 & 7.74 & 7.20 & 9.30 \\
\hline
\end{tabular}

pacientes con afectación en hemisferio izquierdo y hemisferio derecho, se realizó una comparación de medias para cada variable neuropsicológica con la $t$ de Student para muestras independientes. Los resultados indicaron que en ninguna de las variables neuropsicológicas se alcanzó la significación estadística en la comparación entre los pacientes afectados de gliomas en hemisferio derecho e izquierdo (Tabla 12).

\section{Discusión}

Este estudio forma parte de un grupo reducido de investigaciones, que tienen por objetivo determinar, de forma exclusiva, el papel de los gliomas en el rendimiento neuropsicológico de los pacientes, ya que se realiza una valoración exhaustiva con anterioridad al procedimiento quirúrgico. Así, se pretende identificar los aspectos neuropsicológicos de la calidad de vida en pacientes con gliomas, de forma previa a la aplicación de tratamientos oncológicos, corrigiendo algunas de las limitaciones metodológicas citadas a lo largo del texto.

A nivel clínico, los resultados obtenidos arrojan que más de la mitad de la muestra total presenta un rendimiento neuropsicológico por debajo de lo esperado, excepto en orientación y en recuerdo verbal con claves a corto plazo, de acuerdo a las puntuaciones centiles obtenidas por la misma. Este porcentaje es elevado en los dominios de velocidad de procesamiento de la información, memoria visual inmediata y denominación verbal. A pesar de que no era un objetivo prioritario del presente estudio, pueden evidenciarse elevados niveles de malestar emocional, caracterizados por síntomas de ansiedad y depresión, ya que la muestra presenta una puntuación media por encima del punto de corte establecido por los autores de la prueba utilizada para su medición.

De acuerdo a la documentada influencia de la edad y del malestar emocional en el rendimiento neuropsicológico, se controló su efecto en este estudio. Respecto al hemisferio afectado, no se observaron diferencias en las variables neuropsicológicas en 
Tabla 12. Diferencias en las variables neuropsicológicas de acuerdo al hemisferio afectado

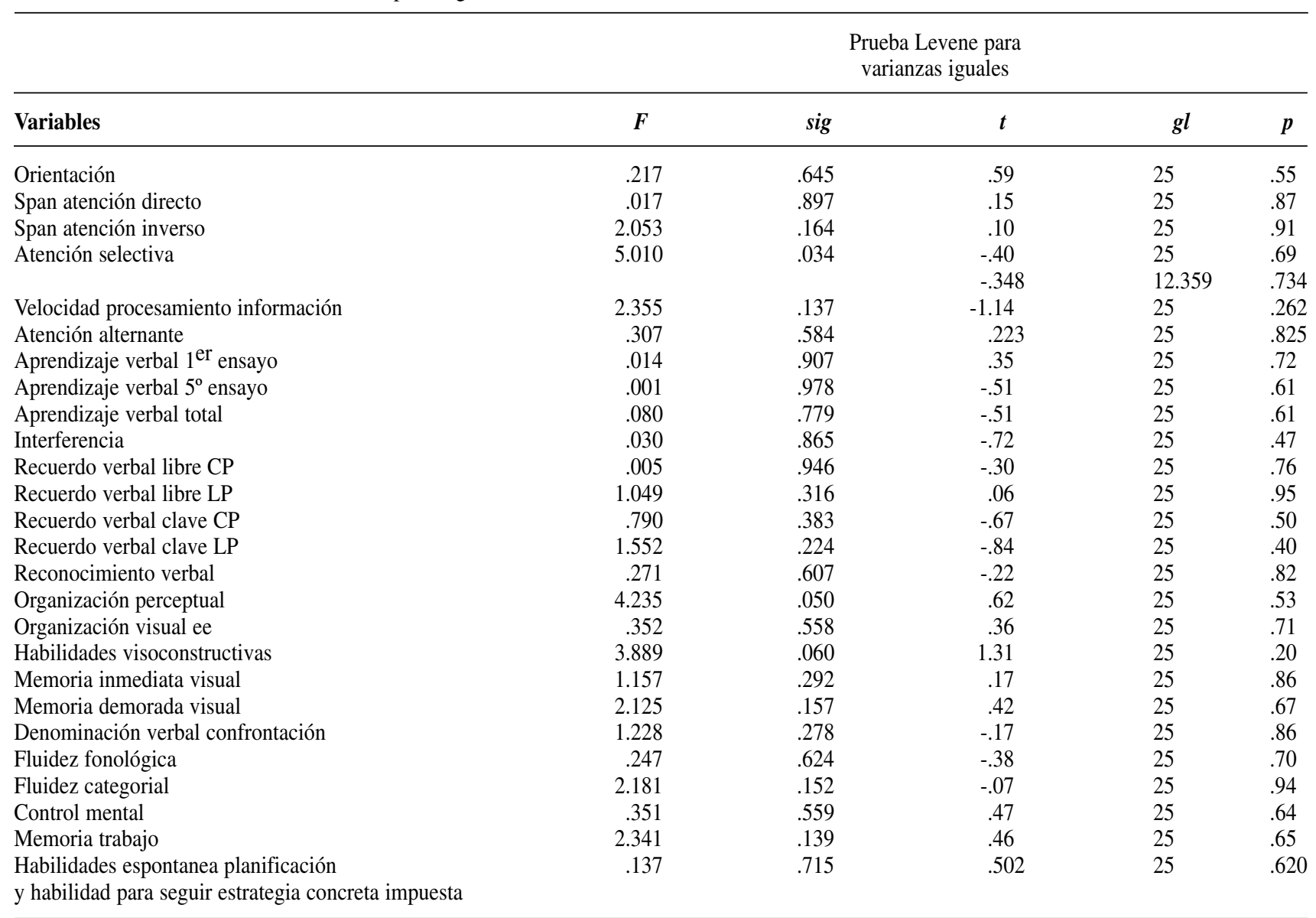

$* \mathrm{p}<.05 ; * * \mathrm{p}<.01 ; * * * \mathrm{p}<.001$

función del mismo, tampoco debido al efecto de la edad. Estos datos coinciden con la línea de resultados obtenidos por Alvarán y cols. (2008), indicando que estos hallazgos pueden deberse al carácter difuso de los déficits neuropsicológicos en los pacientes con tumores cerebrales; hecho que puede producirse por el aumento de la presión intracraneal, el desplazamiento de la línea media, la infiltración del propio tumor, la afectación del volumen sanguíneo circundante a la lesión, la destrucción de tejido sano y la creación de focos de epilepsia.

Nuestros resultados son distintos a los aportados por Tucha y cols. (2000), que sí refieren diferenciación en el rendimiento en memoria verbal y habilidades visoespaciales, en función del hemisferio afectado. La ausencia de coincidencia en los resultados puede deberse a las características de la muestra; en el estudio de Tucha y cols. (2000) existe una hete- rogeneidad en la misma, formada por pacientes con gliomas y metástasis cerebrales. Por el contrario, en nuestro estudio únicamente se seleccionaron pacientes con gliomas; este hecho aporto homogeneidad en la muestra y, además, solvento una de las limitaciones metodológicas más relevantes en este campo, que justifica la discrepancia entre los estudios.

En relación al diagnóstico anatomopatológico, se evidencia de forma exclusiva un mejor rendimiento neuropsicológico en pacientes con gliomas de bajo grado, en un único dominio de funciones ejecutivas (habilidades de planificación y para seguir estrategia concreta impuesta). El resto de las diferencias encontradas en variables neuropsicológicas, tales como atención, memoria, habilidades visoconstructivas, organización visual, lenguaje y funciones ejecutivas, se explican por la influencia de la variable edad y no por el diagnóstico anatomopatológico. 
Estos datos no coinciden con los referidos por Tucha y cols. (2000) y Alvarán y cols. (2008), esta discrepancia puede deberse a que dichos autores no el efecto de la edad en el rendimiento neuropsicológico al comparar entre gliomas de alto y bajo grado. En nuestra investigación, el factor más relevante para explicar las diferencias en este rendimiento entre pacientes con diferente tipo de tumor, es el efecto de la edad sobre el que se ejerció control.

Es por ello, que en futuras investigaciones se deberá aclarar el papel de cada factor en el rendimiento neuropsicológico, de los pacientes con gliomas. Para conseguir este objetivo, sería recomenda- ble considerar la edad, dado que esta variable influye en las puntuaciones de las variables neuropsicológicas, aspecto recogido en la literatura científica y en los datos de este estudio, al explicar las diferencias encontradas en el rendimiento de acuerdo al diagnóstico anatomopatológico. Además, se insta a considerar factores emocionales de ansiedad y depresión elevados, que influyen en el rendimiento neuropsicológico con independencia del hemisferio afectado y del diagnóstico anatomopatológico. Por último, se debería intentar constituir muestras homogéneas, para obtener una mayor especificidad de los resultados, que de estas investigaciones se deriven.

\section{Extended Summary}

In patients with brain tumors, cognitive performance in the study of quality of life is crucial. This is due to the influence of it in different areas of life of patients and its predictive role in the progression of the disease. Actually, patients with gliomas have shown deficits or cognitive impairment along with the disease. It is estimated that over $70 \%$ with this tumor show neurological and/or neuropsychological alterations.

However, studies in this area of Neuro-oncology have a number of methodological limitations regarding to study design, sample size and heterogeneity, as well as at the time of measurement and the neuropsychological instruments applied.

This article describes the results of a neuropsychological assessment in patients with glioma at the time of diagnosis, prior to the application of surgical resection.

Method. The sample included 29 patients (18 men and 11 women) with a mean age of $54,69 \%$ had a pathological diagnosis of high grade glioma (anaplastic astrocytoma and glioblastoma multiforme) and the most common clinical symptoms shown were headache and motor disturbances. 59\% of the sample had the lesion in the right hemisphere, while only $7 \%$ had bilateral lesions. $40 \%$ of all cases showed involvement of the frontal lobe.

The most relevant variables for this study are pathological diagnosis (low-grade gliomas and high grade) and affected hemisphere (right and left), as independent variables. The group of dependent variables consists of the scores on standardized neuropsychological tests involving attention, memory, language, visuoconstructive skills, visual organization, language and executive functions. The control variables in the statistical analysis are the age and emotional state at the time of assessment, as collected using the Hospital Anxiety and Depression Scale Zigmond and Snaith (HADS, 1983).

Results. Over $50 \%$ of the total sample scored below the 40 percentile or below the cutoff, except orientation scale of the WMS-III and the verbal memory task with short-term key TAVEC. This percentage is as much as $80 \%$ in TMT-A, immediate memory task of the Rey Complex Figure and the Boston Naming Test, which involves significant failures in information processing speed, in immediate visual memory and visual confrontation nomination.

The group of patients with low grade glioma showed better performance in planning skills and to further concrete strategy imposed (BADS Zoo Map) $(\mathrm{p}<.001)$. We don't find statistical significance for the remaining neuropsychological variables in accorder the pathologic diagnosis.

In contrast, the age is able to explain the differences in neuropsychological performance between patients with low gliomas and high gliomas in atten- 
tion, memory, language, perceptual organization and executive functions.

Otherwise, there were no differences in neuropsychological performance depending on hemisphere affected by the tumor.

Discussion. This study is part of a small number of investigation that aim to determine the role of gliomas in patient's cognitive performance. We seek to identify the cognitive aspects of quality of life in patients with gliomas, prior to the implementation of cancer treatment, correcting some of the methodological limitations mentioned above.

The results indicated that more than half of the total sample performed below expectations, except in orientation and short-term key verbal memory. This percentage was higher in the domains of information processing speed, immediate visual memory and naming.

Although it wasn't a primary objective of this study, the sample presented high levels of emotional distress, characterized by symptoms of anxiety and depression. The sample had an average score above the cutoff established by the authors of this test.

According to the documented influence of age and emotional distress in neuropsychological performance, its effect was controlled in this study. In regarding to the hemisphere affected, there were no differences in neuropsychological performance of the sample by the tumor, even by the effect of age. These results were similar to those of the study by Alvarán et al. (2008), reporting diffuse nature of neuropsychological impairment in patients with brain tumors.

Contrary to the study by Tucha et al. (2000), whose sample consisted of patients with gliomas and brain metastases, in our study the population consists exclusively of patients with gliomas. This provides consistency in the sample and solves one of the most of important methodological limitations in this area.

In relation to the pathological diagnosis, patients with low-grade gliomas have better neuropsychological performance in a single domain of executive functions (planning skills and to further concrete strategy imposed). The rest of the differences in neuropsychological variables, such as attention, memory, skills visuoconstructive, visual organization, language and executive functions, are explained by the influence of the age and not the pathological diagnosis. These results are not consistent with those reported by Tucha et al. (2000) and Alvarán et al. (2008). This discrepancy may be because these authors didn't consider the effect of age on neuropsychological performance of patients with gliomas.

Therefore, in future research should clarify the role of each factor in the neuropsychological performance of patients with gliomas. To achieve this, it would be advisable to consider the age effect on the scores of the neuropsychological variables. This aspect is reflected in the scientific literature and in data from this study, because the age explains the differences in performance according to pathologic diagnosis. In addition, we recommend considering anxiety and depression, because they influence neuropsychological performance regardless affected hemisphere and pathologic diagnosis. Finally, to obtain greater specificity of results, it should constitute homogeneous samples.

\section{Referencias}

Afra, D., Muller, W. y Benoist, G. (1978). Supratentorial recurrences of gliomas. Acta Neurochirurgica, 43, 217-27.

Alderman, N., Burgess, P. W., Emslie, H., Evans, J. J. y Wilson, B. (1996). BADS - Behavioral Assessment of Dysexecutive Syndrome. London: Thames Valley.

Alvarán, L., Gómez L. A., Aguirre, D. C. y Ortiz, L. D. (2008). Caracterización neuropsicológica de pacientes con glioma tratados en el Instituto de Cancerología de Medellín. Acta Neurológica Colombiana, 24, 13-23.

Álvarez, T. y Conde-Guzón, P. A. (2009). Formación de subtipos de niños con problemas escolares de aprendizaje a partir de la evaluación 
neuropsicológica, capacidades cognitivas y Comportamiento. Clínica y Salud, 20, 19-41.

Armstrong, C. L., Stern, C. H. y Corn, B. W. (2001). Memory performance used to detect radiation effects on cognitive functioning. Applied Neuropsychology, 8, 129-139.

Army Individual Test Battery. (1944). Manual of directions and DC, EEUU: War Department, Adjutant General's Office.

Arrieta, E. y García Alonso, L. M. (2009). Efectividad de las pruebas psicométricas en el diagnóstico y seguimiento de deterioro, depresión y ansiedad en las personas mayores. Clínica $y$ Salud, 2009, 20, 5-18.

Benedet, M. J., y Alejandre, M. A. (1998). TAVEC: Test de aprendizaje verbal España-Complutense. Madrid: TEA.

Benton, A. L. y Hamsher, K. (1989). Multilingual Aphasia Examination, $2^{\text {a }}$ ed. Iowa City: Department of Neurology and Psychology. University of Iowa.

Bondy, M. L., Scheurer, M. E., Malmer, B., Barnholtz-Sloan, J. S., Davis, F. G., Il'yasova, D., ... Buffler, P. A. (2008). On behalf of the Brain Tumor Epidemiology Consortium. Brain Tumor Epidemiology: Consensus from the brain tumor epidemiology consortium. Cancer, 1, 113 (7 Suppl), 1953-1968. doi:10.1002/cncr.23741.

Brown, P., Ballman, K., Rummans, T., Maurer, M. J., Sloan, J. A. y Boeve B. F. (2006). Prospective study of quality of life in adults with newly diagnosed high-grade gliomas. Journal of Neurooncology, 76, 283-291.

Brown, P. D., Buckner, J. C., O'Fallon, J. R., Iturria, N. L., Brown, C. A., O’Neill, B. P., ... Shaw, E. G. (2003). Effects of radiotherapy on cognitive function in patients with low-grade glioma measured by the Folstein Mini-Mental State Examination. Journal of Clinical Oncology, 21, 2519-2524.

De la Cruz, M. V., Seisdedos, N. y Cordero, A. (1997). Test de copia y reproducción de memoria de la figura compleja de Rey. 6 ed. Madrid: TEA.

Douw, L., Klein, M., Fagel, S. S., Van den Heuvel, J., Taphoorn, M. J., Postma, T. J., Vandertop, W.
P., Mojj, J. J., Boerman, R. H., Beute, G. N., Sluimer, J. D., Slotman, B. J., Reijneveld, J. C. y Heimans, J. J. (2009). Cognitive and radiological effects of radiotherapy in patients with low-grade glioma: long-term follow-up. The Lancet Neurology, 8, 810-818.

Gauthier, L., Dehaut, F. y Joanette, Y. (1989). The bells: a quantitavie and qualitative test for visual neglect. International Journal Clinical Neuropsychology, 11, 49-54.

Gregor, A., Cull, A., Traynor, E., Stewart, M., Lander, F. y Love, S. (1996). Neuropsychometric evaluation of long-term survivors of adult brain tumours: Relationship with tumour and treatment parameters. Radiotherapy \& Oncology, 41, 55-59.

Hooper, H. E. (1983). The Hooper visual organization test manual. Los Angeles: Western Psychological Services.

Kaplan, E. F., Goodglass H. y Weintraub, S. (1983). The Boston Naming Test. Philadelphia: Lea y Febiger.

Klein, M., Heimans, J. J., Aaronson, N. K., van der Ploeg, H. M., Grit, J., Muller, M., ... y Taphoorn, M. J. (2002). Effect of radiotherapy and other treatment-related factors on mid-term to long-term cognitive sequelae in low-grade gliomas: a comparative study. Lancet, 360, 1361-1368.

Klein, M., Taphoorn, M. J., Heimans, J., Vander Ploeg, H. M., Vandertup, W. P., Smit, E. F., ... y Aaronson, N. K. (2001). Neurobeha-vioral status and health-related quality of life in newly diagnosed high-grade glioma patients. Journal of Clinical Oncology, 9, 4037-47.

Laack, N. N, Brown, P. D., Ivnik, R. J., Furth, A. F., Ballman, K. V., Hammack, J. E., ... Buckner, J. C. (2005). Cognitive function after radiotherapy for supratentorial low-grade glioma: a North central cancer treatment group prospective study. International Journal of Radiation Oncology Biology Physics, 63, 1175-1183.

Levin, V. A. y Kyristis, A.P. (2000). Gliomas, medulloblastomas, and CNS germ cell tumors. En F Cavalli, H Hansen (Eds.) Textbook of Medical Oncology (pp. 493-520). London: Martin Dunitz, 
Meyers, C. A. y Hess, K. R. (2003). Multifaceted end points in brain tumor clinical trials: Cognitive deterioration precedes MRI progression. Oncology 5, 89-99.

Molina, F., Prujá, E., Vera, R., Marcos, M., Tejedor, M. y Albistur, J. J. (2001). Factores pronóstico en los tumores cerebrales. Anales del Sistema Sanitario de Navarra, 24, 63-72.

Perea, M. V. y Ladera, V. (1995). Rendimientos neuropsicológicos: Edad, Educación y Sexo. Psicothema, 7, 105-112.

Peterson, K. (2001). Brain tumors. Neurologic Clinics, 19, 887-902.

Postma, T. J., Klein, M., Verstappen, C. C., Bromberg, J. E., Swennen, M., Langendijk, J. A., ... y Heimans, J. J. (2002). Radiotherapy-induced cerebral abnormalities in patients with low-grade glioma. Neurology, 59, 121-123.

Reijneveld, J. C., Sitskoorn, M. M., Klein, M., Nuyen, J. y Taphoorn, M. J. (2001). Cognitive status and quality of life in patients with suspected versus proven low-grade gliomas. Neurology, 56, 618-623.

Scheibel, R. S., Meyers, C. A., y Levin, V. A. (1996). Cognitive dysfunction following surgery for intracerebral glioma: influence of histopathology, lesion location, and treatment. Journal of Neurooncology, 30, 61-69. DOI: 10.1007/BF00177444

Surma-aho, O., Niemela, M., Vilkki, J., Kouri, M., Brander, A., Salonen, ... y Jääskeläinen, J. (2001). Adverse long-term effects of brain radiotherapy in adult low-grade glioma patients. Neurology, 56, 1285-1290.

Taphoorn, M. J. (2003). Neurocognitive sequelae in the treatment of low-grade gliomas. Seminars in oncology, 30, 6(Supp 19), 45-48.

Taphoorn, M. J., Schiphorst, A., Snoek, F., Lindeboom, J., Wolbers, J., Karim, A. B., ... y Heimans, J. J. (1994). Cognitive functions and quality of life in patients with low-grade gliomas: the impact of radiotherapy. Annals of Neurology, 36, 48-54.

Torres, I. J., Mundt, A. J., Sweeney, P. J., LlanesMacy, S., Dunaway, L., Castillo, M. y Macdonald, R. L. (2003). A longitudinal neuropsychological study of partial brain radiation in adults with brain tumors. Neurology, 60, 1113-1118.

Tucha, O., Smely, C., Preier, M. y Lange, K. (2000). Cognitive deficits before treatment among patients with brain tumors. Neurosurgery, 47, 324-334.

Vigliani, M. C., Sichez, N., Poisson, M. (1996). A prospective study of cognitive functions following conventional radiotherapy for supratentorial gliomas in young adults: 4-year results. International Journal of Radiation Oncology Biology Physics, 35, 527-533.

Wechsler, D. (1999). Escala de Inteligencia Wechsler para Adultos (WAIS-III) ( $3^{\mathrm{a} E d}$.). Madrid: TEA.

Wechsler, D. (2004). Escala de memoria de Wechsler-III (WMS-III). 3 ed. Madrid: TEA Ediciones.

Yoshii, Y., Tominaga, D., Sugimoto, K., Tsuchida, Y., Hyodo, A., Yonaha, H. y Kushi, S. (2008). Cognitive function of patients with brain tumor in pre and postoperative stage. Surgical Neurology, 69, 51-61.

Zigmond, A. S., Snaith, R. P. (1983). The Hospital Anxiety and Depression Scale. Acta Psychiatrica Scand, 67, 361-370.

Manuscrito recibido: 22/11/2010 Revisión recibida: 24/01/2011 Aceptado: 17/05/2011 